\title{
Extremal problems for convex lattice polytopes: a survey
}

\author{
Imre Bárány
}

\begin{abstract}
What is the minimal area that a convex lattice polygon with exactly $n$ vertices can have? This question, and several similar ones, are considered in this survey.
\end{abstract}

\section{Jarník's result}

We start with a sample problem and its solution. It was in 1926 that Vojtech Jarník [19] posed the following question. Let $\gamma \subset \mathbf{R}^{2}$ be a closed convex curve of length at most $\ell$. Assume that the radius of curvature at every point of $\gamma$ exists, is positive, and is smaller that $7 \ell$. How many lattice points can such a curve $\gamma$ contain? The answer from $[\mathbf{1 9}]$ is that it contains at most

$$
\frac{3}{\sqrt[3]{2 \pi}} \ell^{2 / 3}+O\left(\ell^{1 / 3}\right)
$$

lattice points and that this estimate is best possible. In other words,

$$
\max \left|\gamma \cap \mathbf{Z}^{2}\right|=\frac{3}{\sqrt[3]{2 \pi}} \ell^{2 / 3}+O\left(\ell^{1 / 3}\right),
$$

where the maximum is taken over all convex curves satisfying the above conditions.

Convex lattice polygons appear on the scene instantly: the set

$$
P=\operatorname{conv}\left(\gamma \cap \mathbf{Z}^{2}\right)
$$

is a convex lattice polygon inscribed in $\gamma$ (provided $\gamma$ contains at least 3 lattice points). Thus we can turn the problem around and ask the following. What is the minimal perimeter $\ell$ that a convex lattice polygon with exactly $n$ vertices can have. Formulae 1 and 2 essentially say that

$$
\ell=\frac{\sqrt{6 \pi}}{9} n^{3 / 2}+O\left(n^{3 / 4}\right)
$$

We will see later in Section 8, in a more general setting, how the proof goes.

This is the type of question we are going to discuss in this survey.

2000 Mathematics Subject Classification. Primary 52B20; Secondary 52B60, 11 H06.

Supported in part by Hungarian National Foundation Grants \#62321 and 60427. 


\section{The list of problems}

In this section we state the problems we are going to consider. First we introduce notation and terminology.

A convex body in $\mathbf{R}^{d}$ is a convex compact set with non-empty interior. We write $\mathcal{K}^{d}$ for the family of all convex bodies in $\mathbf{R}^{d}$. As usual, a convex lattice polytope in $\mathbf{R}^{d}$ is the convex hull of finitely many points from the $d$-dimensional integer lattice $\mathbf{Z}^{d}$. The set of $d$-dimensional convex lattice polytopes with nonempty interior will be denoted by $\mathcal{P}^{d}$. Clearly, $\mathcal{P}^{d} \subset \mathcal{K}^{d}$. For a polytope $P \subset \mathbf{R}^{d}$ we write $f_{i}(P)$ for the number of $i$-dimensional faces of $P$. In particular, $f_{0}(P)$ is the number of vertices and $f_{d-1}(P)$ is the number of facets. Finally, $V(K)$ and $S(K)$ stand for the volume and surface area of the convex body $K \in \mathcal{K}^{d}$.

Here comes the list of problems.

Minimal volume. Determine

$$
V_{d}(n)=\min \left\{V(P): P \in \mathcal{P}^{d}, f_{0}(P)=n\right\} .
$$

Minimal surface area. Determine

$$
S_{d}(n)=\min \left\{S(P): P \in \mathcal{P}^{d}, f_{0}(P)=n\right\} .
$$

Minimal lattice width. The width of a convex body $K \in \mathcal{K}^{d}$ in direction $z \in \mathbf{Z}^{d}$ $(z \neq 0)$ is defined as

$$
w(K, z)=\max \{z(x-y): x, y \in K\} .
$$

The lattice width of $K$ (cf. [22] and [20]) is

$$
w(K)=\min \left\{w(K, z): z \in \mathbf{Z}^{d}, \quad z \neq 0\right\} .
$$

The minimal lattice width problem asks for the determination of

$$
w_{d}(n)=\min \left\{w(P): P \in \mathcal{P}^{d}, f_{0}(P)=n\right\} .
$$

We will see later that this question is of little interest.

Arnold's question. In [3] V. I. Arnold posed the following problem. Two lattice polytopes $P, Q \in \mathcal{P}^{d}$ are called equivalent if there is a lattice preserving affine transformation carrying $P$ to $Q$. This is clearly an equivalence relation. Equivalent lattice polytopes have the same volume. Write $N_{d}(V)$ for the number of equivalence classes of lattice polytopes that have volume at most $V$.

Arnold's question was to investigate $N_{d}(V)$ and to determine the order of magnitude of $\log N_{d}(V)$. The problem is related to the number of ways a homogeneous polynomial with $d$ variables can behave near the origin. It is also connected to the minimal volume question as we will soon see.

The question is not an extremal problem on lattice polytopes but is very close to what this survey is about. That is why I treat it here.

Maximal polytopes in $K$. Assume $K \subset \mathbf{R}^{d}$ is "large". Determine

$$
\max \left\{f_{0}(P): P \in \mathcal{P}^{d}, P \subset K\right\} .
$$

This problem, in fact, asks for the maximal size subset of $K \cap \mathbf{Z}^{d}$ which is in convex position. (A set is in convex position if none of its points is contained in the convex hull of the others.) This question is, again, closely related to the minimal volume problem. 
In the next five sections we consider the above problems for all dimensions $d \geq 2$. In every case we find the order of magnitude of the extremum. Asymptotic estimates are available for $d=2$ only (save for the lattice width which is of no interest anyway). In the planar case the asymptotic behaviour of the extrema, and even their limiting shapes, will be explained in the final four sections. This survey contains hardly any new result. Although I have tried to avoid proofs, sometimes I felt a sketch would help the reader in understanding the ideas and the methods. I intended to point out some connections between extremal convex lattice polygons and approximation questions, and affine surface area. There are further connections, for instance to random polytopes and to Sylvester's famous four-point-problem. But this survey is too short to contain them all.

On the technical side we will use the "big Oh" and "little oh" notation, together with Vinogradov's very convenient $\ll$ and $\gg$ symbols. So $f(x) \ll g(x)$ means that there is constant $c$ such that $f(x) \leq c g(x)$ for all values of $x$. This constant of course does not depend on $x$, but does depend on dimension. So, for instance, Theorem 3.1 below means that there is a constant $c_{d}>0$ depending only on $d$ such that $f_{0}(P)^{\frac{d+1}{d-1}} \leq c_{d} V(P)$ for all $P \in \mathcal{P}^{d}$.

There are, of course, several other problems, extremal or otherwise, concerning convex lattice polytopes. Most of them are outside the scope of this survey. The interested reader should consult for instance the thorough survey by Gritzmann and Wills $[\mathbf{1 7}]$ or the beautiful paper by Kannan and Lovász [20]. Some more specific questions are treated in $[\mathbf{1 4}]$ and $[\mathbf{2 1}]$.

\section{Minimal volume}

Of all the problems listed above, that of the minimal volume seems the most natural. The reason is perhaps that it is invariant under lattice preserving affine transformations, and also because its solution helps with the other questions. A lower bound, of the right order of magnitude, was found in a pioneering paper [2] of G. E. Andrews in 1963.

Theorem 3.1. For all $P \in \mathcal{P}^{d}$,

$$
f_{0}(P)^{\frac{d+1}{d-1}} \ll V(P),
$$

where the implied constant depends only on $d$.

The proof is hard. By now there are several other proofs available, by Konyagin and Sevastyanov [23], Schmidt [28], Bárány and Vershik [12], Bárány and Larman [8], and Reisner, Schütt and Werner [27]. All of them are based on different ideas and none of them are simple. We will show later how the answer to Arnold's question implies Andrews's theorem.

The following even more general statement is true. A tower of $P \in \mathcal{P}^{d}$ is a chain of faces, $F_{0} \subset F_{1} \subset \cdots \subset F_{d-1}$, of $P$ with $\operatorname{dim} F_{i}=i$. Write $T(P)$ for the number of towers of $P$. Clearly, $f_{i}(P) \leq T(P)$ for every $i$.

TheOREM 3.2. For all $P \in \mathcal{P}^{d}$

$$
T(P)^{\frac{d+1}{d-1}} \ll V(P)
$$

where the implied constant depends only on $d$. 
This was proved in $[\mathbf{2 3}]$ and [8]. The result implies, of course, that

$$
f_{i}(P)^{\frac{d+1}{d-1}} \ll V(P) \text { for all } i=0,1, \ldots, d-1 .
$$

Actually, Andrews' proof of Theorem 3.1 is based on proving (4) first for $i=d-1$, the facets of $P$.

We mention in passing the following question which is from polytope theory.

Open problem 1. Is there a constant $c_{d}$ depending only on $d$ such that for every polytope $P \in \mathcal{K}^{d}$

$$
T(P) \leq c_{d}\left(f_{0}(P)+f_{1}(P)+\cdots+f_{d-1}(P)\right) .
$$

The bound given in Theorem 3.1 is best possible, apart from the implied constant. This is shown by two examples. The first is due to Arnold [3]. We explain it first in the planar case. Let $P$ be the convex hull of the points $\left(x, x^{2}\right)$ where $x \in \mathbf{Z}$ and $-(n-1) / 2 \leq x \leq(n-1) / 2$ when $n$ is odd, and $-(n-2) / 2 \leq x \leq n / 2$ when $n$ is even. Trivially, $f_{0}(P)=n$. It is very easy to check that the area of $P$ is very close to $n^{3} / 12$.

In higher dimensions $P$ is the convex hull of lattice points on the paraboloid $x_{1}^{2}+\cdots+x_{d-1}^{2}=x_{d}$ restricted to $x_{d} \leq r$. It is simple to see that $f_{0}(P) \gg r^{d-1}$ and $V(P) \ll r^{d+1}$. This shows that the estimate in Theorem 3.1 is of the right order of magnitude for all $d \geq 2$.

The other construction, which is due to Bárány and Larman [8], is equally easy to describe, but proving its properties is harder. Let $B^{d}$ be the Euclidean unit ball of $\mathbf{R}^{d}$ and set

$$
P_{r}=\operatorname{conv}\left(r B^{d} \cap \mathbf{Z}^{d}\right),
$$

where $r$ is a parameter. The polytope $P_{r}$ is the integer convex hull of $r B^{d}$. It is clear that $V\left(P_{r}\right)=r^{d} V\left(B^{d}\right)(1+o(1))$. The next task is to estimate $f_{0}\left(P_{r}\right)$.

THEOREM 3.3 .

$$
f_{0}\left(P_{r}\right) \gg r^{d \frac{d-1}{d+1}}
$$

The proof is based on estimating the volume missed by $P_{r}$.

LEMMA 3.4. $V\left(r B^{d} \backslash P_{r}\right) \ll r^{d \frac{d-1}{d+1}}$.

The proof of the Lemma uses the Flatness Theorem (cf $[\mathbf{2 2}],[\mathbf{2 0}]$ ) saying that the lattice width of a convex body $K \in \mathcal{K}^{d}$ with $K \cap \mathbf{Z}^{d}=\emptyset$ is bounded by a number depending only on $d$.

Next we use a result from the theory of approximation of convex bodies by polytopes. It is well known (cf [18] for instance) that a polytope with $n$ vertices and inscribed in $B^{d}$ misses $\gg n^{-\frac{2}{d-1}}$ volume of $B^{d}$. After suitable scaling we get

$$
\left(f_{0}\left(P_{r}\right)\right)^{-\frac{2}{d-1}} \ll \frac{V\left(r B^{d} \backslash P_{r}\right)}{V\left(B^{d}\right)} \ll r^{d \frac{d-1}{d+1}-d}=r^{-\frac{2 d}{d+1}} .
$$

This implies Theorem 3.3 immediately.

The method works for $f_{i}\left(P_{r}\right)$ as well (by suitably extending the approximation result from vertices to $i$-dimensional faces, see Bárány [6] or Böröczky [15]), and gives

$$
f_{i}\left(P_{r}\right) \gg r^{d \frac{d-1}{d+1}} \text {, for all } i=0,1, \ldots, d-1 .
$$

Of course, $T(P) \geq f_{0}(P)$ implies that $T\left(P_{r}\right) \gg r^{d \frac{d-1}{d+1}}$ as well. 
Remark. The unit ball $B^{d}$ does not play any special role here. Let $K \in \mathcal{K}^{d}$ be a convex body containing 0 in its interior. Assume the boundary of $K$ is smooth and at every point its principal curvatures are separated from 0 and infinity (by constants depending only on dimension). Then the integer convex hull $P_{r}=\operatorname{conv}\left(r K \cap \mathbf{Z}^{d}\right)$ of $r K$ satisfies $f_{i}\left(P_{r}\right) \gg r^{d \frac{d-1}{d+1}}$ for all $i$.

The results in this section show that $V_{d}(n)$, the minimal volume a convex lattice $d$-polytope with $n$ vertices can have, is of order $n^{\frac{d+1}{d-1}}$. The following question is very natural.

Open problem 2. Decide whether

$$
\lim _{n \rightarrow \infty} n^{-\frac{d+1}{d-1}} V_{d}(n)
$$

exists or not. If it does exist, what is its value?

The answer is known only for $d=2$ and is quite surprising (at least for the author). We describe it in Section 9. The case of higher dimensions is beyond reach at the moment; it seems to require significant new ideas.

\section{Surface area}

The isoperimetric inequality says that, for every $K \in \mathcal{K}^{d}$,

$$
\frac{S(K)^{d}}{V(K)^{d-1}} \geq \frac{S\left(B^{d}\right)^{d}}{V\left(B^{d}\right)^{d-1}}
$$

with equality if and only if $K$ is a Euclidean ball. So Theorem 3.1 gives a lower bound for the minimal surface area problem.

Theorem 4.1. For all $P \in \mathcal{P}^{d}$,

$$
f_{0}(P)^{\frac{d}{d-1}} \ll S(P) .
$$

Actually, this was proved by Andrews [1] in 1961, before Theorem 3.1. The estimate is again best possible apart from the implied constant. This is shown by the integer convex hull $P_{r}$. The minimal surface area problem is solved in "order of magnitude" sense:

$$
n^{\frac{d+1}{d}} \ll S_{d}(n) \ll n^{\frac{d+1}{d}} .
$$

Open problem 2. Decide whether

$$
\lim _{n \rightarrow \infty} n^{-\frac{d+1}{d}} S_{d}(n)
$$

exists or not, if it does, what is its value.

The limit exists for $d=2$. By Jarník's classical result (1) the minimal perimeter that a convex lattice $n$-gon can have is $\sqrt{6 \pi} n^{3 / 2} / 9(1+o(1))$. Jarník's method works in a more general setting, when the perimeter is measured by some other norm of $\mathbf{R}^{2}$. We return to this question in Section 8 . 


\section{Lattice width}

This happens to be a question of little interest. First, assume $d=2$ and $P \in \mathcal{P}^{2}$ has exactly $n$ vertices. Let $z \in \mathbf{Z}^{2}$ be the lattice width direction of $P$. By the definition of lattice width exactly $w(P)+1$ lattice lines orthogonal to $z$ intersect $P$. Since every lattice line contains at most two vertices of $P$, we have $n \leq 2(w(P)+1)$, implying

$$
\left\lceil\frac{n}{2}\right\rceil-1 \leq w(P)
$$

The reader will have no difficulty finding a convex lattice polygon with $n$ vertices having lattice width exactly $\lceil n / 2\rceil-1$.

In higher dimension $w(P)=1$ is possible with $f_{0}(P)$ taking arbitrarily large values. Simply take two $(d-1)$-dimensional lattice polytope having together $n$ vertices, one lying in the lattice hyperplane $x_{1}=0$, and the other one in the lattice hyperplane $x_{1}=1$ of $\mathbf{R}^{d}$. The convex hull of these two polytopes is a polytope in $\mathcal{P}^{d}$, of lattice width 1 , with exactly $n$ vertices.

So this problem is either trivial or ill posed. Maybe one should define some kind of "successive lattice widths" and find the proper question.

\section{Arnold's question}

The first result in this direction is due to Arnold. He shows in [3], in the planar case, that

$$
A^{1 / 3} \ll \log N_{2}(A) \ll A^{1 / 3} \log A
$$

as $A$ goes to infinity. We write here $A$, instead of $V$, for the area of a convex lattice polygon. Arnold's proof is based on the estimate in Theorem 3.1 in the planar case (that he found independently of Andrews [2]), and on the square lemma. This lemma states that an aligned square of side length $36 \mathrm{~A}$ contains a convex lattice polygon from each equivalence class with area at most $A$. From this the upper bound $\log N_{2}(A) \ll A^{1 / 3} \log A$ follows easily, since $N_{2}(A)$ is at most the number of ways the $\ll A^{1 / 3}$ vertices can be chosen from the $(36 A)^{2}$ lattice points contained in the square of side length $36 A$.

The $\log A$ factor in the upper bound was removed by Bárány and Pach in [10] showing that the order of magnitude of $\log N_{2}(A)$ is $A^{1 / 3}$.

Arnold's question proved to be fertile, and Konyagin and Sevastyanov [23] proved Theorem 3.1, again independently of Andrews [2]. They used it, together with an extension of the square lemma, to show that

$$
V^{\frac{d-1}{d+1}} \ll \log N_{d}(V) \ll V^{\frac{d-1}{d+1}} \log V .
$$

The extra $\log V$ factor, in every dimension, was removed in [12] by A M Vershik and the present author.

TheOREM 6.1. When $d \geq 2$ and $V \rightarrow \infty$, then

$$
V^{\frac{d-1}{d+1}} \ll \log N_{d}(V) \ll V^{\frac{d-1}{d+1}} .
$$

The proof is based on several ideas from number theory, convexity, and geometry of numbers. It uses the following extension of Arnold's square lemma. First, $\mathbf{Z}_{+}^{d}$ is defined to be the set of all $a=\left(a_{1}, \ldots, a_{d}\right) \in \mathbf{Z}^{d}$ with each $a_{i}>0$. Next, given $z \in \mathbf{Z}_{+}^{d}$, we define the box of $a$ as

$$
\operatorname{Box}(a)=\left\{x \in \mathbf{R}^{d}: 0 \leq x_{i} \leq a_{i}\right\}
$$


For simplicity set $\prod a=\prod_{1}^{d} a_{i}$. Here is the box lemma which replaces the square lemma in the proof.

Lemma 6.2. For every $P \in \mathcal{P}^{d}$ there is an $a \in \mathbf{Z}_{+}^{d}$ with $\prod a \ll V(P)$ such that an equivalent copy of $P$ is contained in $\operatorname{Box}(a)$. The implied constant depends only on $d$.

As the number of boxes with $\prod a \ll V$ is less than $V^{d}$ (actually, much less), the next step in the proof of the upper bound in Theorem 6.1 is the following fact.

Lemma 6.3. For every $a \in \mathbf{Z}_{+}^{d}$ the number of convex lattice polytopes contained in $\operatorname{Box}(a)$ is at most

$$
\exp \left\{c_{d}\left(\prod a\right)^{\frac{d-1}{d+1}}\right\},
$$

where $c_{d}$ is a constant depending only on $d$.

The proof of this lemma is of interest, and we explain its basic idea. Given a polytope $P$ and one of its facets $F$, let $v(F)$ denote the outer normal vector to $P$ at $F$ with length equal to $S(F)$, the $(d-1)$-dimensional volume of $F$. As is well-known, the sum of $v(F)$ for all facets of $P$ is equal to zero,

$$
\sum_{F} v(F)=0
$$

and these vectors linearly span $\mathbf{R}^{d}$. The converse of this fact is known as Minkowski's theorem [14]: Given a system $\left\{v_{1}, \ldots, v_{n}\right\}$ of vectors with these properties, there is a polytope $P$, unique up to translation, with exactly $n$ facets, $F_{1}, \ldots, F_{n}$ say, such that $v\left(F_{i}\right)=v_{i}$.

For a lattice polytope, the vector $v(F)$ satisfies

$$
v(F) \in \frac{1}{(d-1) !} \mathbf{Z}^{d} .
$$

The proof of Lemma 6.3 only uses the fact that each $v(F)$ satisfies this condition and that $P \subset \operatorname{Box}(a)$. It is based on an extension of Minkowski's theorem, due to Pogorelov [25], and an estimate for the number of ways a positive integer vector can be written as a sum of other positive integer vectors.

Remark. There is an interesting issue here. Call a polytope $P \in \mathcal{K}^{d}$ dually integral if condition (5) is satisfied for all facets of $P$. Thus lattice polytopes are dually integral. Theorem 6.1 holds not only for lattice polytopes but for (equivalence classes of) dually integral polytopes of volume at most $V$. Dually integral polytopes seem to behave better than lattice polytopes. In particular, there might be some hope for asymptotic estimates. We mention further that in the planar case lattice polygons and dually integral polygons coincide. I think this is what helps with the asymptotic results for $d=2$.

The proof of the lower bound in Theorem 6.1 is easier. Consider $P \in \mathcal{P}^{d}$ with $n=f_{0}(P) \gg(V(P))^{\frac{d-1}{d+1}}$, for instance $P_{r}$, the integer convex hull of $r B^{d}$. Let $X$ be the set of vertices of $P$. For each nonempty subset $Y$ of $X, \operatorname{conv} Y$ is a convex lattice polytope in $\mathbf{R}^{d}$, of volume at most $V(P)$. There are $2^{n}-1$ such polytopes. It is not hard to see that most of them are $d$-dimensional and very few of them are equivalent. This shows the lower bound in Theorem 6.1.

We remark that this argument can be turned around and used for the proof of Andrews' Theorem 3.1. Suppose that $P \in \mathcal{P}^{d}$ and $V(P) \leq V$. By Lemma 6.2 
we can assume that $P$ lies in some $\operatorname{Box}(a)$ with $\prod a \ll V$. Again, let $X$ be the set of vertices of $P$. For each (nonempty) subset $Y$ of $X$, $\operatorname{conv} Y$ is a convex polytope in the box. These polytopes are all distinct and their number is $2^{|X|}-1$. By Lemma 6.3,

$$
2^{|X|}-1 \leq \exp \left\{c_{d}\left(\prod a\right)^{\frac{d-1}{d+1}}\right\} \ll \exp \left\{c_{d}^{\prime} V^{\frac{d-1}{d+1}}\right\}
$$

with a suitable constant $c_{d}^{\prime}$, implying, in turn, that $|X|=f_{0}(P) \ll V^{\frac{d-1}{d+1}}$.

Theorem 6.1 immediately raises the following problem.

Open problem 4. Decide whether

$$
\lim _{V \rightarrow \infty} V^{-\frac{d-1}{d+1}} \log N_{d}(V)
$$

exists or not. Determine the limit if it exists.

This problem looks hard. The answer is not known even in the planar case. We return to this question in Section 10.

\section{Maximal polytopes in $K$}

We formulate the question slightly differently. Fix $K \in \mathcal{K}^{d}$ and consider the lattice $\mathbf{Z}_{t}=\mathbf{Z}_{t}^{d}=\frac{1}{t} \mathbf{Z}^{d}$ where $t$ is large. In this way we replace the condition " $K$ is large" with " $t$ is large". From now on we may assume that $V(K)=1$. Write $\mathcal{P}(K, t)$ for the family of all convex $\mathbf{Z}_{t}$-lattice polytopes contained in $K$. Here a $\mathbf{Z}_{t}$-lattice polytope is a polytope $P \in \mathcal{K}^{d}$ having all of its vertices in the lattice $\mathbf{Z}_{t}$. Now the question is this. Determine

$$
M(K, t)=\max \left\{f_{0}(P): P \in \mathcal{P}(K, t)\right\}
$$

asymptotically, or up to order of magnitude, as $t \rightarrow \infty . M(K, t)$ is the same as the maximal number of points in $\mathbf{Z}_{t} \cap K$ that are in convex position. The following theorem gives the order of magnitude of $M(K, t)$.

TheOREM 7.1. For all $K \in \mathcal{K}^{d}$ of volume 1 ,

$$
t^{d \frac{d-1}{d+1}} \ll_{K} M(K, t) \ll t^{d \frac{d-1}{d+1}} .
$$

The implied constant in $\ll_{K}$ depends on $K$ as well.

The proof of the upper bound follows from Theorem 3.1. If $P$ is a $\mathbf{Z}_{t}$-lattice polytope in $K$, then $t P$ is an ordinary lattice polytope in $t K$. Thus

$$
f_{0}(P)=f_{0}(t P) \ll \operatorname{Vol}(t P)^{\frac{d-1}{d+1}} \leq \operatorname{Vol}(t K)^{\frac{d-1}{d+1}}=t^{d \frac{d-1}{d+1}},
$$

implying that $M(K, t) \ll t^{d \frac{d-1}{d+1}}$. For the lower bound let $E$ be the maximal volume ellipsoid, inscribed in $K$. Then, according to the remark at the end of Section 3, the integer convex hull of $t E$, that is, $\operatorname{conv}\left(t E \cap \mathbf{Z}^{d}\right)$ has the right number of vertices. The dependence on $K$ only shows up in the fact that $t^{d \frac{d-1}{d+1}} \ll M(K, t)$ begins to be true for larger and larger values of $t$, as the body $K$ gets more and more flat, or needle-like.

The asymptotic behaviour of $M(K, t)$ is only known in the planar case. We describe it in Section 11. 


\section{Minimal perimeter}

From now on we are going to work in the planar case. We fix the extra notation needed. A nonzero vector $z \in \mathbf{Z}^{2}$ is called primitive if its components are relatively prime. Let $\mathbf{P} \subset \mathbf{Z}^{2}$ be the set of all primitive vectors in $\mathbf{Z}^{2}$. It is wellknown that the density of $\mathbf{P}$ in $\mathbf{Z}^{2}$ is $6 / \pi^{2}$. We will need more precise estimates related to this fact. They are best formulated in the following simple lemma that has been known, in several different forms, for ages.

LEMMA 8.1. Assume $D \in \mathcal{K}^{2}$ contains the origin in its interior. Suppose $f: \mathbf{R}^{2} \rightarrow \mathbf{R}$ is a homogeneous map of degree $\alpha \geq 0$, that is, $f(\lambda x)=\lambda^{\alpha} f(x)$ for every $x \in \mathbf{R}^{2}$ and every $\lambda \geq 0$, and $|f(x)| \leq 1$ for all $x \in D$. Then

$$
\sum_{z \in \lambda D \cap \mathbf{P}} f(z)=\lambda^{\alpha+2}\left\{\frac{6}{\pi^{2}} \int_{D} f(x) d x+O\left(\lambda^{-1} \log \lambda\right)\right\} .
$$

Back to minimal perimeter now. The question is answered by Jarník's result (3) or (2). In fact, Jarník's method works in a more general setting. Namely, let $D \in \mathcal{K}^{2}$ be the unit ball of a (not necessarily symmetric) norm in $\mathbf{R}^{2}$. We only assume that the centre of gravity of $D$ is at the origin, that is,

$$
\int_{D} z d z=0
$$

(with vector integration). As usual, the norm, or rather $D$-norm, of a vector $z \neq 0$ is

$$
|z|=|z|_{D}=\max \left\{t^{-1}: t z \in D, t>0\right\} .
$$

For $P \in \mathcal{P}^{d}$, let $x_{1}, \ldots, x_{n}$ denote the vertices of $P$ along the perimeter in anticlockwise direction. Set $z_{i}=x_{i+1}-x_{i}$ for $i=1, \ldots, n$, where $n+1$ is identified with 1 . The perimeter of $P$ when measured by the $D$-norm is

$$
S_{D}(P)=\sum_{1}^{n}\left|z_{i}\right|
$$

The minimal $D$-perimeter problem asks for the determination of

$$
S_{D}(n)=\min \left\{S_{D}(P): P \in \mathcal{P}^{2}, f_{0}(P)=n\right\} .
$$

The solution, due to M Prodromou [26], follows Jarník's method with minor modifications. (For the $\ell_{p}$ norm this was proved by Stojakovic [30] earlier.)

THEOREM 8.2. Under the above conditions

$$
S_{D}(n)=n^{\frac{3}{2}}\left(\frac{\pi \int_{D}|z| d z}{\sqrt{6(\text { Area } D)^{3}}}+O\left(n^{-1} \log n\right)\right) .
$$

Proof. We only give a sketch. Assume $P \in \mathcal{P}^{2}$ has $n$ vertices, and let $z_{1}, \ldots, z_{n}$ be its edge-vectors as described above. Suppose that $p_{1}, \ldots, p_{n}$ are the shortest (in $D$-norm) $n$ vectors in $\mathbf{P}$. In case of ambiguity, any $n$ shortest vectors will do. Let $\lambda>0$ be the smallest number with

$$
\left\{p_{1}, \ldots, p_{n}\right\} \subset \lambda D .
$$

Thus all $\left|p_{i}\right| \leq \lambda$. Let $U$ be the set of primitive vectors in $\lambda D$, distinct from the $p_{i}$. Clearly, $|u|=\lambda$ for each $u \in U$. Further, $|U|=O(\lambda)$ as the boundary of $\lambda D$ 
contains at most $O(\lambda)$ lattice points. Thus, by Lemma 8.1 with $f(z) \equiv 1$,

$$
n=|\lambda D \cap \mathbf{P}|-|U|=\frac{6}{\pi^{2}} \lambda^{2} \text { Area } D\left(1+O\left(\lambda^{-1} \log \lambda\right)\right) .
$$

This is the asymptotic relation between $n$ and $\lambda$.

We clearly have

$$
\sum_{1}^{n}\left|z_{i}\right| \geq \sum_{1}^{n}\left|p_{i}\right|=\sum_{p \in \mathbf{P} \cap \lambda D}|p|-\lambda|U|
$$

Here $\lambda|U|=O\left(\lambda^{2}\right)$, and the sum preceding it can again be estimated by Lemma 8.1 with $f(z)=|z|$ :

$$
\sum_{p \in \mathbf{P} \cap \lambda D}|p|=\frac{6}{\pi^{2}} \lambda^{3} \int_{D}|z| d z\left(1+O\left(\lambda^{-1} \log \lambda\right)\right.
$$

Plugging in the value of $\lambda$ as a function of $n$ gives the lower bound in Theorem 8.2.

The next task is to construct a lattice polygon with $n$ vertices and the same perimeter. The $n$ shortest primitive vectors almost produce such a polygon: the trouble is that their sum is not zero. But it is almost zero! Indeed, summing the first and second components of the $p_{i}=\left(x_{i}, y_{i}\right)$ separately we get, using again Lemma 8.1 with $f(z)=x$ where $z=(x, y)$, that

$$
\sum_{1}^{n} x_{i}=\sum_{p=(x, y) \in \lambda D} x+O\left(\lambda^{2}\right)=\frac{6}{\pi^{2}} \lambda^{3}\left(\int_{D} x d z+O\left(\lambda^{-1} \log \lambda\right)\right)=O\left(\lambda^{2} \log \lambda\right)
$$

since $\int_{D} x d z=0$ because 0 is the centre of gravity of $D$. The same works for the $y$ component. Thus $p_{0}=\sum_{1}^{n} p_{i} \in \mathbf{Z}^{d}$ has $D$-norm $O\left(\lambda^{2} \log \lambda\right)$, and, of course, $-p_{0}+p_{1}+\cdots+p_{n}=0$. So the vectors $-p_{0}, p_{1}, \ldots, p_{n}$, as edges, form a unique convex lattice polygon, with $n+1$ edges (or $n$ edges if $-p_{0}=\alpha p_{i}$ for some $p_{i}$ and $\alpha>0$.) This polygon has the right $D$-perimeter:

$$
\left|-p_{0}\right|+\sum_{p \in \mathbf{P} \cap \lambda D}|p|=\frac{6}{\pi^{2}} \lambda^{3} \int_{D}|z| d z\left(1+O\left(\lambda^{-1} \log \lambda\right) .\right.
$$

If it has $n+1$ edges, then replacing two consecutive edges by their sum produces the required convex lattice $n$-gon.

Remark. The idea of the above proof is that, instead of the extremal polygon, one should look for the edge set of the extremal polygon. As we will soon see, the same approach works in other problems as well.

I mention further that Theorem 8.2 is slightly stronger than the one in Prodromou [26] because she only considers norms with 0 -symmetric unit ball.

Theorem 8.2 can be further strengthened because the minimizer $P_{n}$, that is, the convex lattice $n$-gon with minimal perimeter, is almost completely determined by the norm. After suitable normalization $P_{n}$ tends to a fixed convex set, $P \in \mathcal{K}^{2}$, which can be explicitly described. A convenient way to explain this uses the support function $h_{K}: S^{1} \rightarrow \mathbf{R}$ of $K \in \mathcal{K}^{2}$. This is defined by $h_{K}(u)=\max \{u \cdot x: x \in K\}$; see [14], for instance. As is wellknown, the support function $h_{K}$ determines the convex body $K$ uniquely. 
THEOREM 8.3. Assume $P_{n}$ is a minimizer for $D$ translated so that its center of gravity lies at the origin. Then

$$
\lim _{n \rightarrow \infty} n^{-\frac{3}{2}} h_{P_{n}}(u)=\frac{\pi}{2 \sqrt{6(\text { Area } D)^{3}}} \int_{D, u \cdot x \geq 0} u \cdot x d x .
$$

It follows easily that $h(u)=\lim n^{-\frac{3}{2}} h_{P_{n}}(u)$ is the support function of a convex set $P \in \mathcal{K}^{2}$, and $P$ is the limit shape of the sequence $P_{n}$ after suitable normalization.

\section{Minimal area}

For simpler notation we set $A(n)=V_{2}(n)$. In this section we explain, following Bárány and Tokushige $[\mathbf{1 1}]$, why $\lim n^{-3} A(n)$ exists and is, most likely, equal to $0.0185067 \ldots$

THEOREM 9.1. $\lim _{n \rightarrow \infty} n^{-3} A(n)$ exists.

We only give a sketch. A polygon $P_{n} \in \mathcal{P}$ with $n$ vertices is called a minimizer if Area $P_{n}=A(n)$. Let $\mathcal{K}_{0}$ denote the family of all 0 -symmetric convex bodies in $\mathbf{R}^{2}$. Given $C \in \mathcal{K}_{0}$, set

$$
A^{*}(C)=\frac{1}{8} \sum_{x, y \in C \cap \mathbf{P}}|\operatorname{det}(x, y)| .
$$

We consider the following extremal problem.

$$
\operatorname{Min}(n)=\min \left\{A^{*}(C): C \in \mathcal{K}_{0} \text { and }|C \cap \mathbf{P}|=n\right\}
$$

The first step in the proof is the following

Claim 9.2. For even $n \geq 3, A(n)=\operatorname{Min}(n)$.

For the proof of this claim one notes first that each edge of a minimizer $P_{n}$ is a primitive vector. Next one shows that, for even $n$, there is always a centrally symmetric minimizer, $P_{n}$, say. The edges of $P_{n}$ are then the vectors $z_{1}, \ldots, z_{k},-z_{1}, \ldots,-z_{k}$, where $n=2 k$. It is clear that

$$
A(n)=\operatorname{Area}\left(P_{n}\right)=\sum_{1 \leq i<j \leq k}\left|\operatorname{det}\left(z_{i}, z_{j}\right)\right| .
$$

Set $C_{n}=\operatorname{conv}\left\{ \pm z_{1}, \ldots, \pm z_{k}\right\}$. One proves next that $C \cap \mathbf{P}=\left\{ \pm z_{1}, \ldots, \pm z_{k}\right\}$. It is simple to check that $A^{*}\left(C_{n}\right)=$ Area $P_{n}$.

So we have reduced our problem from lattice polygons to the minimization problem $\operatorname{Min}(n)$. Note that this reduction is fully in the spirit of the remark in the previous section: we work with the edge set of the minimizer, rather than with the minimizer itself.

Observe next that the solution $C$ to the problem $\operatorname{Min}(n)$ is invariant under lattice preserving linear transformations. Thus we may fix $C$ in standard position. This means that the lattice width of $C$ is $2 b=2 b(C)$ and is taken in direction $(0,1)$. Let $[-a, a]$ (where $a=a(C)$ ) be the intersection of $C$ with the $x$ axis. We assume further that the tangent line to $C$ at $(a, 0)$ has slope at least 1 ; this is the case when $C$ is in standard position.

We are going to find the solution $C$ to $\operatorname{Min}(n)$ in standard form. But first we give two examples that will be illustrative and helpful. 
Example 1. Set $C=\operatorname{conv}\{ \pm(0,1), \pm(1,1), \ldots, \pm(k-2,1)\}$. Apart from lattice preserving linear transformations, this is the unique $C \in \mathcal{K}_{0}$ with $b(C)=1$ and $|C \cap \mathbf{P}|=n$ where $n=2 k$. It is easy to see that $A^{*}(C)=\frac{1}{48} n^{3}(1+o(1))$.

Example 2. Set $C=r B^{2}$ with $r>0$ chosen so that $|C \cap \mathbf{P}|=n$. One shows readily that $A^{*}(C)=\frac{1}{54} n^{3}(1+o(1))$.

It is generally expected that $|C \cap \mathbf{P}|=\frac{6}{\pi^{2}}$ Area $C(1+o(1))$. But this is not true when $C$ is thin, for instance in Example 1. One can show (see [11]), however, using the same standard methods that lead to Lemma 8.1, that

$$
|C \cap \mathbf{P}|-\frac{6}{\pi^{2}} \text { Area } C \mid \ll \text { Area } C \cdot \frac{\log b(C)}{b(C)} .
$$

A little more effort (cf $[\mathbf{1 1}])$ gives

$$
\left|8 A^{*}(C)-\left(\frac{6}{\pi^{2}}\right)^{2} \int_{C} \int_{C}\right| \operatorname{det}(x, y)|d x d y| \ll(\text { Area } C)^{3} \cdot \frac{\log b(C)}{b(C)} .
$$

Remark. The last two inequalities measure how far the density of $\mathbf{P}$ in $C$ is from the expected $6 / \pi^{2}$, and measure it as a function of a lattice invariant of $C$, namely $b(C)$. I think this is the crucial step in the proof of Theorem 9.1.

Busemann's theorem [16] states that, among all $K \in \mathcal{K}_{0}$ with fixed area, the integral $\int_{C} \int_{C}|\operatorname{det}(x, y)| d x d y$ is the smallest for the Euclidean ball (or any other ellipse) of the same area. This theorem, together with the last two inequalities, easily implies the following.

Corollary 9.3. Assume $b \geq 2$. Then

$$
A^{*}(C) \geq\left(\frac{1}{54}-D \frac{\log b(C)}{b(C)}\right) n^{3}
$$

where $D$ is a universal constant.

The assumption $b \geq 2$ is justified because the case $b=1$ is completely covered in Example 1, which, as we have seen, is not minimal.

Consider a minimizer, $C_{n}$, for $\operatorname{Min}(n)$. Set $b_{n}=b\left(C_{n}\right)$ and $a_{n}=a\left(C_{n}\right)$. If $b_{n} \rightarrow \infty$, then, by the corollary, $\liminf n^{-3} A(n) \geq 1 / 54$ which, together with Example 2 would give that $\lim n^{-3} A(n)=1 / 54$. Can it happen, though, that $b_{n}$ does not go to infinity?

Yes, it can, and this is where the solution lies. If $b_{n}$ does not tend to infinity, then $b_{n}=b$ along a subsequence $n^{\prime}$ for some $b \in \mathbf{Z}^{2}$. Let $H_{n}$ be the $2 \times 2$ diagonal matrix with diagonal elements $1 / a_{n}$ and 1 . The sequence of convex bodies $H_{n^{\prime}} C_{n^{\prime}}$ is bounded. Consequently it contains a convergent subsequence, with limiting convex body $C^{*}$, say. Define the $2 \times 2$ diagonal matrix $G_{n}$ with diagonal elements $\lambda_{n}$ and 1 , where $\lambda_{n}>0$ is chosen so that $G_{n} C^{*}$ contains exactly $n$ primitive vectors. Setting $C_{n}^{*}=G_{n} C^{*}$ it is not hard to check that

$$
\lim \frac{A^{*}\left(C_{n^{\prime}}\right)}{n^{\prime 3}}=\lim \frac{A^{*}\left(C_{n}^{*}\right)}{n^{3}} .
$$

This shows that whenever we have a sequence of minimizers $C_{n^{\prime}}$, we can find a full sequence $C_{n}^{*}, n=4,6,8, \ldots$ for which $A^{*}\left(C_{n}^{*}\right) / n^{3}$ has the same limit as $A^{*}\left(C_{n}^{\prime}\right) / n^{\prime 3}$.

The last limit depends only on $C^{*}$, which, in turn, depends only on the $4 b$ real numbers given by the $x$-components of the points where the lines $y=i$ intersect the 
boundary of $C^{*}$. This number can be reduced to $b$ by showing that $C^{*}$ is symmetric with respect to the coordinate axes. Suppose now that $C^{*}$ intersects the line $y=i$ in the interval $\left[-x_{i}, x_{i}\right], i=1, \ldots, b$. Thus $C^{*}$ is determined by the parameters $x_{1}, \ldots, x_{b}$. The $x_{i}>0$ form a decreasing and convex sequence. One can express $\lim A^{*}\left(C_{n}^{*}\right) / n^{3}$ as a function of the $x_{i}$ :

$$
\lim \frac{A^{*}\left(C_{n}^{*}\right)}{n^{3}}=\frac{f\left(x_{1}, \ldots, x_{b}\right)}{\left(4 \sum_{i=1}^{b} \frac{\phi(i)}{i} x_{i}\right)^{3}},
$$

where $f\left(x_{1}, \ldots, x_{b}\right)=\sum_{i=1}^{b} \sum_{j=1}^{i} \alpha_{i j} x_{i} x_{j}^{2}$ and the coefficients $\alpha_{i j}$ depend on $i$ and $j$ and $\phi$ is Euler's totient function. This leads to a minimization problem, one for every $b=2,3, \ldots$ :

$$
\begin{aligned}
& \text { minimize } f\left(x_{1}, \ldots, x_{b}\right) \text { subject to } 4 \sum_{i=1}^{b} \frac{\phi(i)}{i} x_{i}=1, \\
& x_{1}, \ldots, x_{b}>0 \text { is decreasing and convex. }
\end{aligned}
$$

The minimum, which clearly exists, is denoted by $M(b)$. The limit in Theorem 9.1 is not $1 / 54$, if a single $M(b)$ is smaller than $1 / 54$. It turns out that $M(8)$ and $M(9)$ are smaller, the smallest that was found is $M(15)=0.0185067 \ldots$ This is smaller than $1 / 54=0.0185185 \ldots$ by about $10^{-5}$. Numerical computations show that $M(b)$ oscillates around $1 / 54$ in ever smaller waves. Luckily, Corollary 9.3 implies that for large enough $b, M(b)$ cannot stay below $M(15)$. The constant $D$ in Corollary 9.3 is about 5000 which gives the following theorem:

THEOREM 9.4. $\lim A(n) / n^{3}=\min _{b \leq 10^{10}} M(b)$.

The exact determination of the right hand side might be possible, although there are $10^{10}$ extremum problems to solve. This number can be reduced further to $10^{7}$ which is still far too many. Computational evidence (cf. [11]) suggests however that $M(b)$ is minimal at $b=15$. This leads to the next open problem which is a computational, and most likely solvable, question.

Open problem 5. Determine $\min _{b \leq 10^{10}} M(b)$.

Remark. Busemann's theorem suggests that the minimizer $C_{n}$ is close to an ellipse. This indeed seems to be the case: $C_{n}$ is close to an ellipse with short axis of length 15.5, and long axis of length $0.0125 n$. The resulting minimizer $P_{n}$ is close to a very oblong ellipse, whose half axes are of lengths $1.656 n$ and $0.00357 n^{2}$. The minimizers $C_{n}$ have a limiting shape, namely $C^{*}$, but only after one-sided normalization. Again, there is a limit shape to the minimizers $P_{n}$ after shrinking in direction $x$ by a factor of $n^{2}$ and in direction $y$ by a factor of $n$.

\section{Arnold's question: the planar case}

In the spirit of Arnold's question it is natural to ask how many convex lattice polytopes there are in $\operatorname{Box}(a)$. The planar version of this question will be considered in the present section. We set up the problem a little differently. Let $K \in \mathcal{K}^{2}$ be a convex body, and recall the definition of $\mathcal{P}(K, t)$, which is the family of all convex $\mathbf{Z}_{t}$-lattice polygons that are contained in $K$. How many such polygons are there, and what do they look like? This beautiful and inspiring question is due to A M Vershik. 
It comes as a pleasant surprise that $\log |\mathcal{P}(K, t)|$ can be determined quite precisely. The following result is from [5].

TheOREM 10.1. For every $K \in \mathcal{K}$

$$
\lim _{t \rightarrow \infty} t^{-2 / 3} \log |\mathcal{P}(K, t)|=3 \sqrt[3]{\frac{\zeta(3)}{4 \zeta(2)}} A^{*}(K),
$$

where $A^{*}(K)$ is the maximal affine perimeter that a convex body contained in $K$ can have.

See Blaschke [13], Lutwak [24] or Bárány [5] for the definition and properties of affine perimeter. It is shown in [5] that there is a unique convex body $K_{0} \subset K$ whose affine perimeter equals $A^{*}(K)$. The correspondence $K \mapsto K_{0}$ gives a map $\mathcal{K} \rightarrow \mathcal{K}$ which is affinely equivariant, that is, for every nondegenerate affine transformation $L,(L K)_{0}=L\left(K_{0}\right)$. The unique $K_{0}$ has interesting properties. For instance, it contains no line segments on its boundary, and its boundary is a parabola arc whenever it lies in the interior of $K$. See [10] for further properties of $K_{0}$.

The convex polygons in $\mathcal{P}(K, t)$ have a limit shape: almost all of them are very close to $K_{0}$. This is the content of the next theorem, where $\delta(X, Y)$ denotes the Hausdorff distance of $X, Y \subset \mathbf{R}^{2}$.

TheOREM 10.2. For every $K \in \mathcal{K}$ and every $\varepsilon>0$,

$$
\lim _{t \rightarrow \infty} \frac{\left|\left\{P \in \mathcal{P}(K, t): \delta\left(P, K_{0}\right)<\varepsilon\right\}\right|}{|\mathcal{P}(K, t)|}=1 .
$$

This theorem was first proved in the case when $K$ is the unit square. Then $K_{0}$ is bounded by four parabola arcs, each touching consecutive edges of the unit square at their midpoints. This case was proved by Vershik [31] and Bárány [4]. Sinai [29] gave a probabilistic proof, together with a central limit theorem on the distribution of the convex $\mathbf{Z}_{t}$-lattice polygons lying in the vicinity of $K_{0}$. The case of general $K \in \mathcal{K}$ comes from [5]. We mention that this limit shape result is much stronger than the one in Theorem 8.3; there are very few minimizers there for a fixed $n$, while here the majority of the $\exp \left\{c t^{2 / 3}\right\}$ convex lattice polygons are very close to $K_{0}$.

More recently, Vershik and Zeitouni [32] established central limit theorems about how big a fraction of $\mathcal{P}(K, t)$ is close to $K_{0}$.

We now return to Arnold's question when $d=2$. The constant in the exponent is still not known:

Open problem 6. Decide whether

$$
\lim _{A \rightarrow \infty} A^{-\frac{1}{3}} \log N_{2}(A)
$$

exists or not. Determine the limit if it exists.

This is just a repetition of Open problem 4 for $d=2$, but there is some hope here. As we have seen in the proof of Theorem 6.1 for every convex lattice polygon of area at most $A$, there is an equivalent lattice polygon contained in $\operatorname{Box}(a, b)=\left\{(x, y) \in \mathbf{R}^{2}: 0 \leq x \leq a, 0 \leq y \leq b\right\}$ with $a b \leq 6 A$. (This is the planar version of the box lemma, with explicit constant 6 .)

Write $N(a, b)$ for the number of convex lattice polygons in $\operatorname{Box}(a, b)$. Which box of area $6 A$ contains the largest number of convex lattice polygons? As $a, b$ go 
to infinity with their ratio bounded, the answer follows from Theorem 10.2:

$$
N(a, b)=\exp \left\{6 \sqrt[3]{\frac{2 \zeta(3)}{\zeta(2)}} \sqrt[3]{a b}(1+o(1))\right\} .
$$

It turns out that the same asymptotic formula is valid as long as $a, b$ go to infinity satisfying $b \leq a$ and $a=o\left(b^{2}\right)$. Moreover, $N(a, b)$ becomes much less than that when $a \geq \varepsilon_{0} b^{2}$ for a fixed small $\varepsilon_{0}>0$. An interesting thing happens when $a=\varepsilon b^{2}$ and $b \rightarrow \infty$ and $\varepsilon$ is small. Namely, the constant in the exponent changes to

$$
6 \sqrt[3]{\frac{2 \zeta(3)}{\zeta(2)}}+h(\varepsilon)
$$

where $h(\varepsilon)$ tends to zero and oscillates around zero infinitely often as $\varepsilon \rightarrow 0$. This observation, which is due to Gábor Halász (see [4]), shows that the maximum $N(a, b)$ when $a b \leq 6 A$ behaves a little strangely. This phenomenon is similar to the behaviour of the minimal area convex lattice $n$-gon. We close this section with

Open problem 7. Determine $\varepsilon>0$ for which $h(\varepsilon)$ is maximal.

\section{Maximal polygons in $K$}

In the planar case, $M(K, t)$ can be determined asymptotically; see Bárány and Prodromou [10].

THEOREM 11.1. Under the above conditions,

$$
\lim _{t \rightarrow \infty} t^{-2 / 3} M(K, t)=\frac{3}{(2 \pi)^{2 / 3}} A^{*}(K)
$$

Here $A^{*}(K)$ is, again, the maximal affine perimeter a convex body inscribed in $K$ can have. As we know, $A P\left(K_{0}\right)=A^{*}(K)$ for a unique convex body $K_{0} \subset K$. This unique $K_{0}$, again, has the limit shape property.

THEOREM 11.2. Let $Q_{t}$ denote any maximizer in the definition of $M(K, t)$. Then

$$
\lim _{t \rightarrow \infty} \delta\left(Q_{t}, K_{0}\right)=0
$$

where $\delta$ denotes the Hausdorff distance.

This fact need not be surprising, especially after Theorem 10.2. There is another connection worth explaining: the affine perimeter governs the approximability of a smooth convex set $K \in \mathcal{K}^{2}$ in the following sense. Consider the best approximating polygon $Q_{n} \in \mathcal{P}$, inscribed in $K$, with $n$ vertices. Best here means that Area $\left(K \backslash Q_{n}\right)$ is the smallest for $Q_{n}$ among all such polygons. If $K$ has $\mathcal{C}^{2}$ boundary, then

$$
\text { Area }\left(K \backslash Q_{n}\right)=\frac{1}{12} A P(K)^{3} n^{-2}(1+o(1)) \text {. }
$$

So $K_{0}$ is the worst approximable convex subset of $K$ in the sense that it requires the largest number of vertices for $Q_{n}$ in order to approximate it within $\varepsilon$ say, that is, to achieve Area $\left(K \backslash Q_{n}\right)<\varepsilon$. Then one would expect many $\mathbf{Z}_{t}$-lattice points in a small neighbourhood of the boundary of $K_{0}$. That may be one reason why $K_{0}$ is actually the limiting shape for maximal polygons (Theorem 11.2), and also why the overwhelming majority of the polygons in $\mathcal{P}(K, t)$ lie in a small neighbourhood of $K_{0}$ (Theorem 10.2). 
Interestingly, Theorem 11.2, or rather its proof, can be used for a characterization of $K_{0}$. Write $\mathcal{C}$ for the set of those $C \in \mathcal{K}$ whose centre of gravity is at the origin, that is, $\int_{C} z d z=0$. The radial function of $C, \rho_{C}$ is a map from $S^{1} \rightarrow R$ such that for a unit vector $u \in S^{1} \rho_{C}(u)=\max \{t>0: t u \in C\}$. The condition $\int_{C} z d z=0$ translates (via a change of variables to polar coordinates) to

$$
\int_{S^{1}} \rho(u)^{3} d u=0
$$

here $d u$ denotes vector integration on $S^{1}$. By Minkowski's classical theorem (see [14]), there is a unique (up to translation) convex body $C^{*}$ whose radius of curvature in direction $u$ is exactly $R(u)=\frac{1}{3} \rho^{3}(u)$. The following characterization theorem, which is due to Bárány and Prodromou [10], describes the sets $K_{0}$ when $K \in \mathcal{K}^{2}$.

TheOREM 11.3. For each $K \in \mathcal{K}^{2}$, there is a unique $C \in \mathcal{C}$ such that $K_{0}$ is a translated copy of $C^{*}$. Moreover, for every $C \in \mathcal{C}$ the set $C^{*} \in \mathcal{K}^{2}$ has the largest affine perimeter among all convex sets contained in $C^{*}$, that is, $\left(C^{*}\right)_{0}=C^{*}$.

This theorem immediately implies the following result.

Corollary 11.4. Assume $K \in \mathcal{K}^{2}$. Then $K_{0}=K$ holds if and only if $K$ has well defined and continuous radius of curvature $R(u)$ (for each $u \in S^{1}$ ) and $\sqrt[3]{3 R(u)}$ is the radial function of a convex set $C \in \mathcal{C}$.

I find it quite remarkable that the motivation and proof method for a theorem in ordinary convex geometry come from considering convex lattice polygons.

Acknowledgement My thanks are due to Gergely Ambrus, Jürgen Eckhoff, and Peter McMullen for thorough reading of this paper and useful comments on its content.

\section{References}

[1] Andrews, G.E., Asymptotic expression for the number of solutions of a general class of Diophantine equations. Trans. AMS, 99 (1961), 272-277.

[2] Andrews, G.E., A lower bound for the volumes of strictly convex bodies with many boundary points. Trans. AMS, 106 (1963), 270-279.

[3] Arnold, V.I., Statistics of integral convex polytopes,Funk. Anal. Pril., 14 (1980), 1-3 (in Russian). English translation: Funct. Anal. Appl., 14 (1980), 79-84.

[4] Bárány, I., The limit shape of convex lattice polygons, Discrete Comp. Geom., 13 (1995), 270-295.

[5] Bárány, I., Affine perimeter and limit shape, J. reine angew. Math., 484 (1997), 71-84.

[6] Bárány, I., The technique of $M$-regions and cap-coverings: a survey, Rendiconti di Palermo, 65 (2000), 21-38.

[7] Bárány, I., Füredi, Z., The lattice diameter of a convex polygon, Discrete Math., 241 (2001), 41-50.

[8] Bárány, I., Larman, D.G., The convex hull of the integer points in a large ball, Math. Annalen, 312, 167-181 (1998).

[9] Bárány, I., Pach, J., On the number of convex lattice polygons, Combinatorics, Probability, and Computation 1 (1992), 295-302.

[10] Bárány, I., Prodromou, M., On maximal convex lattice polygons inscribed in a plane convex set, Israel J. Math., 154 (2006), 337-360.

[11] Bárány, I., Tokushige, H., The minimum area convex lattice $n$-gon, Combinatorica, 24 (2004), 171-185.

[12] Bárány, I., Vershik, A. M., On the number of convex lattice polytopes, GAFA Journal, 2 (1992), 381-393. 
[13] Blaschke, W., Vorlesungen über Differentialgeometrie II. Affine Differentialgeometrie, Berlin, Springer, (1923)

[14] Bonnesen, T., Fenchel, W., Theorie der konvexen Körper, Springer, 1974.

[15] Böröczky, K. Jr, Polytopal approximation bounding the number of $k$-faces, J. Approx. Theory, 102 (2000), 263-285.

[16] Buseman, H., Volume in terms of concurrent cross-sections, Pacific J. Math., 3 (1953), 1-12.

[17] Gritzmann, P., Wills, J. M., Lattice points, in: Handbook of convex geometry, North-Holland (1993) 765-797.

[18] Gruber, P.M., Aspects of approximation of convex bodies, in: Handbook of Convex Geometry, North Holland (1993), 319-345.

[19] Jarník, V., Über Gitterpunkte in konvexen Kurven. Math. Zeit., 24 (1926), 500-518.

[20] Kannan, R., Lovász, L., Covering minima and lattice point free convex bodies, Annals of Math., 128 (1988), 577-622.

[21] Kantor, J-M., On the width of lattice-free simplices. Compositio Math., 118 (1999), 235-241.

[22] Khintchin, A., A quantitative formulation of Kronecker's theory of approximation, Izv.Akad. Nauk SSSR, Ser. Mat., 12 (1948), 113-122 (in Russian).

[23] Konyagin, S.B., Sevastyanov, S.V., Estimation of the number of vertices of a convex integral polyhedron in terms of its volume, Funk. Anal. Pril., 18 (1984), 13-15 (in Russian). English translation: Funct. Anal. Appl., (1984)18, 11-13.

[24] Lutwak, E., On the semicontinuity of curvatures, Comment. Math. Helv., 67 (1992), 664669 .

[25] Pogorelov A. V., Exterior geometry of convex surfaces, (in Russian), Nauka, Moscow, 1969.

[26] Prodromou, M., Limit shape of convex lattice polygons with minimal perimeter, Discrete Math., 300 (2005), 139-151.

[27] Reisner, Sh., Schütt, C., Werner, E., Dropping a vertex or a facet from a polytope, Forum Math., 13 (2001), 359-378.

[28] Schmidt, W., Integral points on surfaces and curves, Monatshefte Math., 99 (1985), 45-82.

[29] Sinai, Ya. G., Probabilistic approach to analyse the statistics of convex polygonal curves, Funk. Anal. Appl. 28 (1994), 41-48 (in Russian). English translation: Funct. Anal. Appl., 28 (1994), 108-113.

[30] Stojaković, M., Limit shape of optimal convex lattice polygons in the sense of different metrics, Discrete Math., 271 (2003), 235-249.

[31] Vershik, A. M., The limit shape for convex lattice polygons and related topics, Funk. Anal. Appl., 28 (1994), 16-25 (in Russian). English translation: Funct. Anal. Appl., 28 (1994), $13-20$.

[32] Vershik A. M., Zeitouni, O., Large deviation in the geometry of convex lattice polygons, Israel J. Math., 109 (1999), 13-27.

Rényi Institute of Mathematics, Hungarian Academy of Sciences, POBox 127, 1364 Budapest, Hungary, and Department of Mathematics, University College London, Gower Street, LONDON WC1E 6BT, UK

E-mail address: barany@renyi.hu 